\title{
Controlling self-organized criticality in sandpile models
}

\author{
Daniel O. Cajueiro ${ }^{1}$ and R. F. S. Andrade ${ }^{2}$ \\ ${ }^{1}$ Department of Economics, Universidade de Brasília, DF 70910-900, Brazil \\ ${ }^{2}$ Instituto de Física, Universidade Federal da Bahia, BA 40210-340, Brazil
}

(Received 19 August 2009; revised manuscript received 13 November 2009; published 19 January 2010)

\begin{abstract}
We introduce an external control to reduce the size of avalanches in some sandpile models exhibiting self-organized criticality. This rather intuitive approach seems to be missing in the vast literature on such systems. The control action, which amounts to triggering avalanches in sites that are near to become critical, reduces the probability of very large events, so that energy dissipation occurs most locally. The control is applied to a directed Abelian sandpile model driven by both uncorrelated and correlated depositions. The latter is essential to design an efficient and simple control heuristic, but has only small influence in the uncontrolled avalanche probability distribution. The proposed control seeks a trade-off between control cost and large event risk. Preliminary results hint that the proposed control works also for an undirected sandpile model.
\end{abstract}

DOI: $10.1103 /$ PhysRevE.81.015102

PACS number(s): 05.65.+b, 02.30.Yy, 45.70.Ht, 89.75.Da

Since the seminal ideas of self-organized criticality (SOC) [1] were applied to a simple sandpile model, this concept has evolved to describe a much larger number of systems such as earthquakes [2], evolutionary bursts [3], forest fires [4], rice piles [5], and financial markets [6]. In SOC dynamics, energy is injected at a constant low rate while dissipation occurs in avalanchelike events of different sizes.

Human beings have always attempted to understand and control nature. Within the SOC framework, "control" can be understood as a series of man-devised actions to interfere in the processes by which the system dissipates energy, in such a way as to concentrate dissipation in moderate sized events and reduce the occurrence probability of very large avalanches. The difficulties to control large events such as earthquakes, hurricanes, floods, and so on depend both on the magnitude of the stored energy as well as on the impossibility of interfering, in an appropriate way, in the dynamics of energy dissipating events. However, under certain limits, other events following the SOC statistics can be subjected to human control. In particular, there are studies that deal with the engineering problem of inducing snow avalanches in restricted hill slides [7], where the purpose is to warrant safety for ski riders. Although not explored yet, similar control may reduce crisis caused by the break of large economic bubbles, which arise due to asymmetry of information or speculative behavior [8].

In this work, we show how it is possible to reduce the risk associated with the occurrence of large avalanches in a SOC system, by considering the simplest directed Abelian sandpile model proposed by Dhar and Ramaswamy [9]. The control scheme, devised to avoid large avalanches in a preselected restricted area of the system, is divided into two different stages. In the first one there is no direct intervention in the system. The control just learns about the dynamics of the system and acquires a global estimate of avalanche risk in the preselected area. In the second stage, which starts when such knowledge has been achieved, the control scans the preselected region and identifies potentially large events whenever the avalanche risk is high enough. Once a threat is detected, an externally induced avalanche is triggered. The scanning phase has a large cost of CPU time and computer operations during numerical simulations, but it does not modify the model rules. Changes in the rules are restricted to the intervention phase. The devised control heuristic takes into account a simple balance between cost and risk represented by large events.

The research area on control strategies applied to specific outputs of a complex system is of increasing interest. The classical example is the chaos control methodology $[10,11]$. Other examples may be found in complex social systems [12], complex biological networks [13], communication systems [14], and discharge plasmas [15].

The Dhar model [9] considers a two-dimensional square lattice of $N \times N$ sites $(i, j)$, with $i, j=1, \ldots, N$. Each site stores a certain amount $z_{i j}$ of mass units. At each time step, the system is driven by two update rules: (a) addition rule: at each time step, a mass unit is added to a randomly selected site $(k, \ell)$, so that $z_{k \ell} \rightarrow z_{k \ell}+1$. (b) Toppling rule: if $z_{i j}>z_{c}$ $=1$, then $z_{i j} \rightarrow z_{i j}-2, z_{i+1, j} \rightarrow z_{i+1, j}+1$, and $z_{i, j+1} \rightarrow z_{i, j+1}+1$. The model is usually represented after performing a $5 \pi / 4$ rotation of the standard square lattice, in such a way the site $(i+1, j+1)$ lies just below the site $(i, j)$, and the $\mathbf{x}$ and $\mathbf{y}$ directions are at $5 \pi / 4$ and $7 \pi / 4$ angles with the horizontal axis.

To speed up the avalanche size control, we may change the nature of the mass deposition process. It amounts to consider a weighted deposition probability similar to the one presented in [16]: if at time $t$, a particle was deposited on the site $(i, j)$, the probability to select the site $(k, \ell)$ to add the particle at $t+1$ is

$$
P[(k, \ell) /(i, j)]=\frac{A}{\{\delta[(i, j),(k, \ell)] / B\}^{\gamma}},
$$

where $\delta[(i, j),(k, \ell)]$ is the Euclidian distance between sites $(i, j)$ and $(k, \ell)$, while $A$ and $B$ are constants related to the normalization of $P$ and to the largest distance between any two sites on the system. The uncorrelated scenario corresponds to choosing $\gamma=0$ and $A=1 / N^{2}, B=1$. The correlated deposition rules can be justified by the existence of a natural time correlation in rain, snow, social, and financial events. The model was implemented both for $\gamma=0$ and $\gamma>0$. Although this change is not essential to the results, uncorrelated 
deposition causes a noticeable additional tracking cost. The size control also takes advantage of the fact that the model is directed.

It is convenient to distinguish between SOC systems that require the presence of a "carrier" for the event propagation (trees in forest-fire models) from those which do not (actual sand or snow avalanches). Control mechanisms are more easily to be implemented on actual system with carriers. Although Dhar's model assumes essential sandpile features, it requires the presence of an excess mass along the avalanche path, or the event dies out. Thus, the model dynamics is, in a certain sense, similar to those for systems with carriers. On the other hand, models that aim to describe systems without such a feature, e.g., sliding snow avalanches with increasing size, may not be suitable to be controlled along the proposed lines.

In order to implement a useful strategy, it is necessary to select a target size $a_{c}$, which is a choice for the largest natural avalanche that might occur in the system. Of course $a_{c}>1$; otherwise, we would have to release down hill the added mass grain at each time unit. Therefore, consider the two-dimensional system $\Gamma$ schematically represented by the array

$$
\Gamma=\left[\begin{array}{ccccccc}
O & O & O & O & O & O & O \\
O & O & O & O & O & O & O \\
O & O & T_{L} & T & T_{R} & O & O \\
O & O & L & X & R & O & O \\
O & O & B_{L} & B & B_{R} & O & O \\
O & O & O & O & O & O & O \\
O & O & O & O & O & O & O
\end{array}\right] .
$$

In Eq. (2), each element of $\Gamma$ indicated by $O, T_{L}, T, T_{R}, L, X$, $R, B_{L}, B$, and $B_{R}$ represents by itself a fixed size square region of sites, corresponding to smaller arrays of order $N_{R} \times N_{R}$. We assume here that avalanche size control takes place inside the region $X$ only. $O$ indicates all matrix positions that are not in the Moore neighborhood of $X . B, L, R$, and $T$ label the following neighboring positions with respect to $X$ : bottom, left, right, and top. They play a special role in our study, as they may trigger or just propagate avalanches that reach the region $X$. They can bounce back or simply be influenced by avalanches triggered inside $X$. The model dynamics is uniform over the whole lattice, so that sites on the border of any region may receive (deliver) grains from (to) the neighboring region.

A number of steps are required to control the avalanche sizes inside $X$. An avalanche in $X$ may arise when the deposition process adds a particle in a site belonging to this region (internal avalanche) or as consequence of an avalanche that started in another region of the system $\Gamma$ (external avalanche). The mathematical modeling of this process (at least in finite scale, far from the thermodynamic limit) is not simple since it is based on a larger set of coupled stochastic nonlinear difference equations.

Let $\mathcal{R}$ be the set of all regions in the system $\Gamma$. In the first control stage, one has to estimate the conditional probability $p_{K / J}(t+1 / t)$ of occurring the addition of mass in region
$K \in \mathcal{R}$ at time $t+1$ assuming that mass was added on a site in region $J \in \mathcal{R}$ at time $t$. In the second stage, such estimates lead to the definition of a threshold value $p_{c}$ that decides whether the control should be activated whenever a new mass unit is deposited in a given region of $\Gamma$. If at time $t$ the mass is added on the region $J \in \mathcal{R}$ and $p_{X / J}(t+1 / t) \geq p_{c}$, then the control should be activated. Such an activation requires one to check the effect of adding a unit mass at any of the sites in the controlled region $X$, i.e., to follow any virtual avalanche that would occur inside the region $X$ if any of the sites in $X$ were actually chosen at random. In order to follow the virtual avalanches, we consider an internal replica $\Gamma_{X}$ of the system, i.e., a restricted copy of the model that describes its dynamics inside the region $X$, as if it was isolated from the rest of $\Gamma$. Based on this replica of $X$, if any added particle in site $(i, j) \in X$ generates a virtual avalanche of size $a \geq a_{c}$, the control "explodes" the corresponding site of $\Gamma$. This means that a real avalanche is triggered by emptying the site $(i, j)$, which amounts to topple the single unit mass with $50 \%$ of probability to the site $(i+1, j)$ or to the site $(i, j+1)$.

For instance, let $X$ be given as follows:

$$
\left[\begin{array}{lllll}
0 & & 1 & & 0 \\
& 1 & & 1 & \\
0 & & 1 & & 0 \\
& 0 & & 0 &
\end{array}\right],\left[\begin{array}{lllll}
0 & & 0 & & 0 \\
& 0 & & 1 & \\
1 & & 0 & & 0 \\
& 1 & & 1
\end{array}\right],\left[\begin{array}{ccc}
X_{R} & \\
0 & 0 & 0 \\
1 & 0 & \\
0 & 0 & 1 \\
1 & 1
\end{array}\right] .
$$

Assume that the control is activated with $a_{c}=3$. Then, the control scans the region $X$ seeking for danger of great avalanches. Note that the only occupied site that may trigger an avalanches larger than $a_{c}$ is the one at the first line, while the occupied sites at the second and third lines are not dangerous. Therefore, the control explodes the critical site and, depending on the side grain topples, one may find one either the configuration $X_{L}$ (if the grain topples leftward) or $X_{R}$ (if in the other direction). In this example, in no other situation the control would intervene in the system.

A fundamental point here is that the size of the virtual avalanche observed in $\Gamma_{X}$ is only a lower bound estimation of the actual avalanches that take place in $X$. First, the replica considers a priori that the internal avalanches are entirely contained in $X$, not considering the influence that these avalanches may receive from their Moore neighbors. Second, the restricted model $\Gamma_{X}$ clearly does not consider the external avalanches that may be triggered in other areas of the system $\Gamma$ and reach $X$. The balance between cost and risk considers that our control is devised to avoid large avalanches within $X$ and that, due to the large size of $\Gamma$, it would be unacceptably expensive to propose a scheme to follow all possible avalanches over the entire system.

The control cost is measured by the number of sites that were accessed to verify whether they are saturated or not and by the number of explosions that have been carried out. If a site is saturated, it is necessary to assess the size of the possible "virtual" avalanche. This requires the cost of simulating the event for any site $(i, j) \in X$ that could become critical. For the correlated process (1) and an appropriate threshold value $a_{c}$, the cost control can be reduced. Indeed, for a 


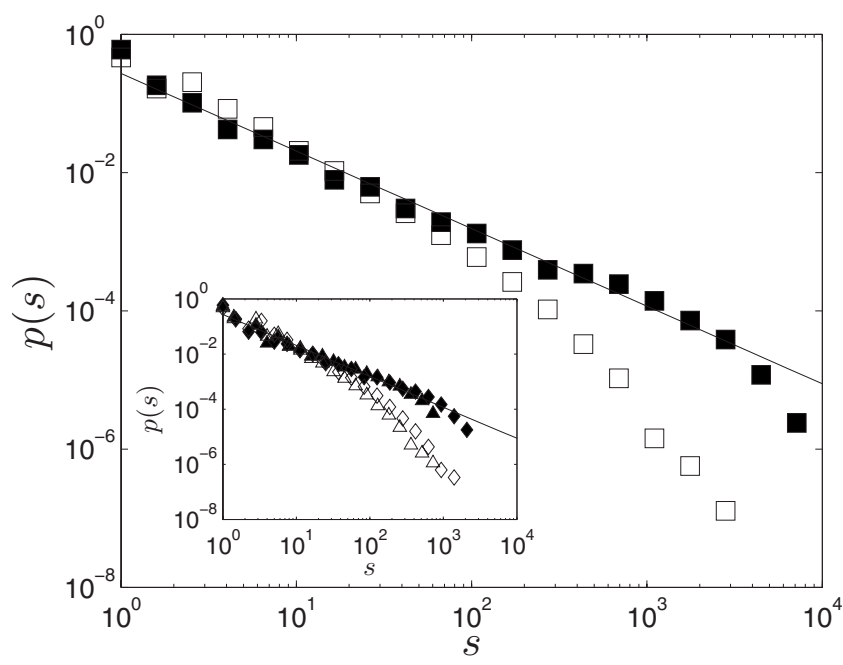

FIG. 1. Probability distribution of avalanche sizes $p(s)$ in region $X$ when $N_{R}=128$. Points were obtained by logarithmic size bins over the whole range of $s$. In the inset, curves are for $N_{R}=64$ (diamonds) and 32 (triangles). Solid and hollow symbols denote uncontrolled and controlled systems, respectively. Both straight lines result from least-square fitting to the solid symbols in the main panel. $a_{c}=4$ for all three cases.

strongly correlated deposition process, there is only a small probability that it will add particles in the region $X$ at $t+1$ if a unit mass was added to any of the other 40 regions of system $\Gamma$ that is not in the Moore neighborhood of $X$ at time $t$.

The choice of $p_{c}$ is based on the following robust heuristic based on the neighbors of the region $X$ :

$$
\begin{aligned}
p_{c}= & \min \left[p_{X / T_{L}}(t+1 / t), \quad p_{X / T}(t+1 / t),\right. \\
& p_{X / T_{R}}(t+1 / t), \quad p_{X / L}(t+1 / t), \\
& p_{X / R}(t+1 / t), \quad p_{X / B_{L}}(t+1 / t), \\
& \left.p_{X / B}(t+1 / t), \quad p_{X / B_{R}}(t+1 / t)\right] .
\end{aligned}
$$

Equation (3) was implemented in systems with $7 \times 7$ regions [see Eq. (2)], with $N_{R}=32,64$, and 128, and $\gamma=1$ in Eq. (1). Figure 1 compares the probability distribution function (PDF) of avalanche sizes $p(s)$ of the uncontrolled system (solid symbols) with that of the controlled system (hollow symbols). Note that $s$ counts only the number of sites in $X$ that topple during the event and, correspondingly, $p(s)$ identifies avalanches where at least one toppling site belongs to $X$. Thus, in a given event, $s$ can be smaller than the total number $s^{*}$ of toppling sites in the whole system. While the data of the uncontrolled system include internal and external avalanches, those of the controlled system include internal, external, as well as avalanches triggered by the control system. The PDF of the uncontrolled systems seems to follow a power law $p(s) \sim s^{-\tau_{c}}$ with an exponent $\tau_{c} \approx 1.12$, while the PDF obtained for the original Dhar model is described by an exponent $\tau=4 / 3$. The straight line in Fig. 1 is the best fit to the data for the system with $N_{R}=128$ in the interval $s \in\left[10^{0}, 10^{3.6}\right]$. It is clear from Fig. 1 that the same exponent

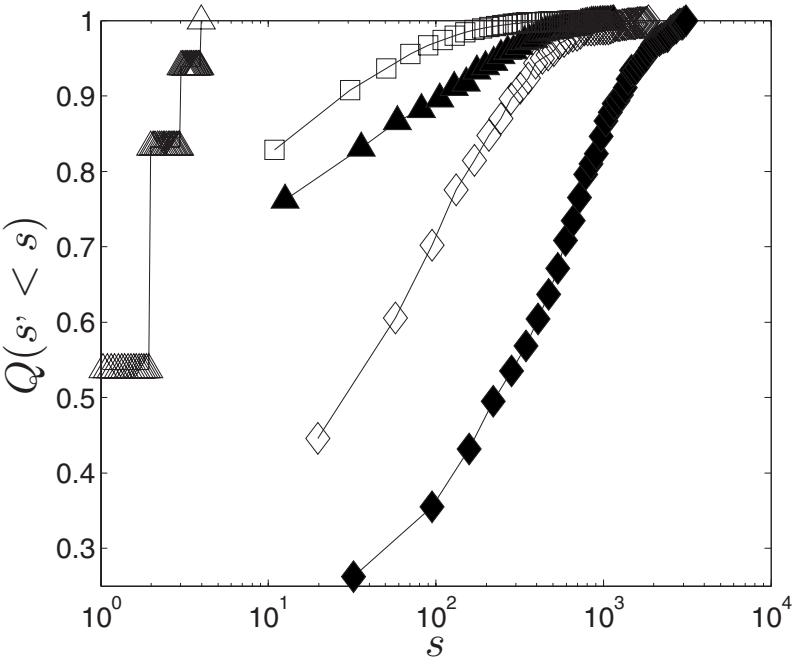

FIG. 2. Complementary probability $Q\left(s^{\prime}<s\right)$ when $N_{R}=64$ and $a_{c}=4$. Solid (hollow) symbols refer to uncontrolled (controlled) system: triangles, diamonds, and squares indicate internal, external, and control generated avalanches.

holds for the systems with $N_{R}=32$ and 64 as their slopes are roughly the same as for $N_{R}=128$. Finite-size effects are made evident by the position of the last two points, which deviate from the straight line. Figure 1 also shows that the introduced control is able to strongly reduce the probability of large events. Graphs with similar features are obtained when $a_{c}=8$.

In Fig. 2, we show the complementary probabilities $Q_{u}\left(s^{\prime}<s\right)=\int_{0}^{s} p_{u}\left(s^{\prime}\right) d s^{\prime}$, where $p_{u}(s)$ describes the specific avalanche distribution types, i.e., $u$ indicates internal, external, or control induced (explosion) avalanches. Figure 2, where each individual $p_{u}(s)$ is normalized to 1 , shows clearly the effect of the external control on the size and on the type of avalanches. Although we use the $\Gamma_{X}$ replica to follow possible avalanches, the control is efficient to reduce both internal and external avalanches.

Figure 3 evaluates the efficiency of the control system by the ratio $f$ between the numbers of avalanches of the controlled to the uncontrolled system. It makes clear that the control system is actually reducing the number of large size avalanches, i.e., its effect is not restricted to increasing the number of small and medium size events. In Fig. 3, we also illustrate the effect of increasing $a_{c}$. It is intuitive that, if $a_{c}$ is increased, the controller is less efficient to reduce the chance of large avalanches, but larger values $a_{c}$ are clearly more economical. This can be seen in the small $s$ region in Fig. 3, where the number of small avalanches of the controlled system with $a_{c}=8$ is smaller than that with $a_{c}=4$. To be more precise, $a_{c}=4$ and 8 require, respectively, 0.32 and 0.12 interventions per time. In fact, the computational cost of the scanning phase was empirically determined to have the same order of growth as $N_{R}^{2}$ and to have a smaller order of growth than $a_{c}$. Moreover, we have empirically found that the number of interventions has a smaller order of growth than $N_{R}$ and decreases almost linearly with $a_{c}$.

The inset in Fig. 3 shows the performance of the random control, which scans the system with the same frequency of 


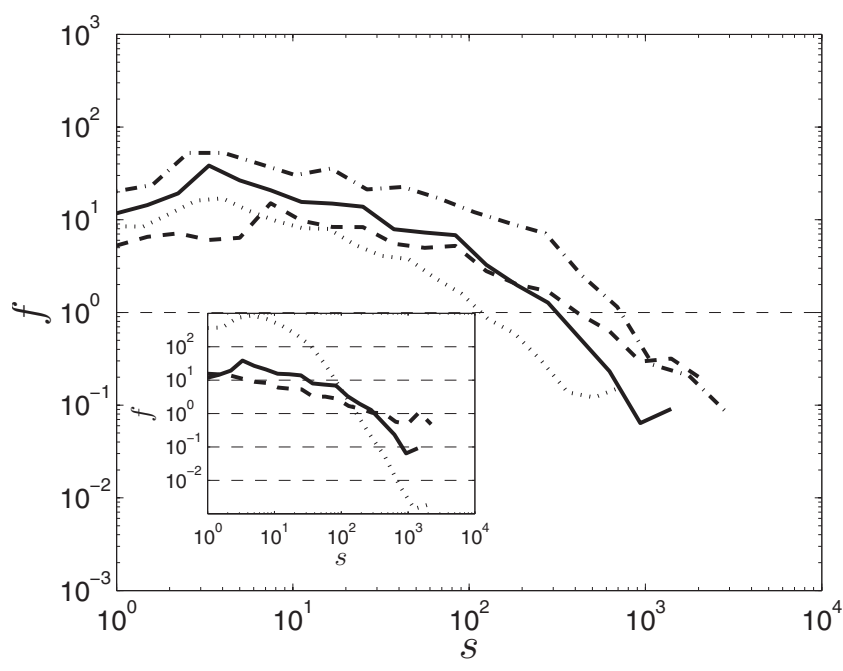

FIG. 3. Ratio $f$ between total number of avalanches in the controlled and uncontrolled simulations. The number of time steps (equal for both simulations) depend on the system size. Line types indicate the following values of $\left(N_{R}, a_{c}\right)$ : $(64,4)$, solid curve; $(64,8)$, dashed curve; $(32,4)$, dotted curve; and $(128,4)$, dotted-dashed curve. Decrease in $f$ in the large size region indicates control success. The increase in the value of $f$ for very large avalanche sizes, observed for some curves, is due to finite-size effects. The logarithmic scale in vertical axis shows that, in the inset, the performance of random control (dashed curve) is much worse that that provided by targeted control (solid curve). The inset also displays results for the BTW model, with $\left(N_{R}, a_{c}\right)=(32,4)$ (dotted curve), showing that the control works also very efficiently.

targeted control and blindly explodes some saturated sites. The slight decrease in the number of large avalanches results from the fact that, since only saturated sites are exploded by random process, some of them are correctly chosen. However, the random control performs much worse for any values of $N_{R}$ and $a_{c}$.

It is still worth commenting that, in the uncorrelated deposition process, the transition probability from any region $I \in \Gamma$ to any region $J \in \Gamma$ is the same and, in our case, given by $1 / 49$. If $p_{c}>1 / 49$, the controller will never scan the system, but if $p_{c}<1 / 49$ it will do at each time step and, contrary to what we observed above, the scanning cost would be very high. Our simulations have shown that controlling a process with correlated or uncorrelated deposition presents a very similar performance in the risk reduction, as long as $p_{c}$ is sufficiently small. This happens because both of them will intervene only when there is a risk of virtual avalanche larger than $a_{c}$.

Although we have considered Dhar's model as a starting point for studying the problem of controlling systems that exhibit SOC, results from preliminary simulations in Fig. 3 suggest that these ideas also work for undirected systems such as the Bak-Tang-Wiesenfeld (BTW) model [1]. In that case, the undirected nature of the model causes a much larger number of small and medium size explosion avalanches.

Our results show that a simple control system reduces the risk of large avalanches in SOC models. Interesting paths to be followed are (1) to propose a control scheme that does not depend on the simulated virtual avalanches, but only on some properties of the internal structure of the system that can be used as early-warning signals $[17,18]$, and (2) to apply this scheme to real SOC systems such as the one presented in [19].

The authors thank CNPq (Brazilian agency) and the National Institute of Science and Technology for Complex Systems for financial support.
[1] P. Bak, C. Tang, and K. Wiesenfeld, Phys. Rev. Lett. 59, 381 (1987).

[2] C. H. Scholz, The Mechanics of Earthquakes and Faulting (Cambridge University Press, Cambridge, England, 1991).

[3] P. Bak and K. Sneppen, Phys. Rev. Lett. 71, 4083 (1993).

[4] B. Drossel and F. Schwabl, Phys. Rev. Lett. 69, 1629 (1992).

[5] V. Frette, K. Christensen, A. Malthe-Sorenssen, J. Feder, T. Jossang, and P. Meakin, Nature (London) 379, 49 (1996).

[6] M. Bartolozzi, D. B. Leinweber, and A. W. Thomas, Physica A 370, 132 (2006).

[7] D. McClung and P. Schaerer, The Avalanche Handbook (The Mountaineers, Seattle, WA, 1993).

[8] J. M. Harrison and D. M. Kreps, Q. J. Econ. 92, 323 (1978).

[9] D. Dhar and R. Ramaswamy, Phys. Rev. Lett. 63, 1659 (1989).

[10] E. Ott, C. Grebogi, and J. A. Yorke, Phys. Rev. Lett. 64, 1196 (1990).
[11] A. Hübler, Helv. Phys. Acta 62, 343 (1989).

[12] D. O. Cajueiro and W. L. Maldonado, Phys. Rev. E 77, 035101(R) (2008).

[13] R. Chakrabarti, H. Rabitz, S. L. Springs, and G. L. McLendon, Phys. Rev. Lett. 100, 258103 (2008).

[14] S. Hayes, C. Grebogi, E. Ott, and A. Mark, Phys. Rev. Lett. 73, 1781 (1994).

[15] W. X. Ding, H. Q. She, W. Huang, and C. X. Yu, Phys. Rev. Lett. 72, 96 (1994).

[16] T. Ohtsuki and H. Iwata, Phys. Rev. A 45, 2228 (1992).

[17] O. Ramos, E. Altshuler, and K. J. Maloy, Phys. Rev. Lett. 102, 078701 (2009)

[18] M. Scheffer, J. Bascompte, W. A. Brock, V. Brovkin, S. R. Carpenter, V. Dakos, H. Held, E. H. VanNes, M. Rietkerk, and G. Sugihara, Nature (London) 461, 53 (2009).

[19] E. Altshuler, O. Ramos, C. Martinez, L. E. Flores, and C. Noda, Phys. Rev. Lett. 86, 5490 (2001). 